\title{
Enseñanza artística universitaria: una mirada desde la relación con el saber
}

\author{
Magalí Pastorino ${ }^{1}$ \\ Ano María Fernóndez Caraballo²
}

\section{Resumen}

La enseñanza artística universitaria local del arte visual se configura en los años sesenta a partir, fundamentalmente, de las concepciones de sujeto y saber que proponían las escuelas experimentales. Sin embargo, el efecto de las actividades experimentales en la enseñanza supera el marco psicologicista e instala un territorio inaudito por su carácter espectacular, lúdico y festivo.

En este sentido, buscamos revisar cómo surgen dichas concepciones, así como analizar la condición de novedad de las llamadas experiencias en la enseñanza del arte visual. La noción relación con el saber —en la vía de Beillerot y su equipo de investigación de la Universidad Paris $\mathrm{X}$, proveniente del psicoanálisis lacaniano— nos brindará una mayor comprensión acerca de esta enseñanza.

Palabras claves: enseñanza artística universitaria, experiencia, relación con el saber, sujeto, arte visual

\begin{abstract}
The local university artistic teaching of visual art is configured in the sixties from, fundamentally, the conceptions of the subject and knowledge of the experimental schools. However, the effect of experimental activities in teaching goes beyond the psychologic framework and establishes an unprecedented territory due to its spectacular, playful and festive character.

In this sense, we seek to review the conception of the subject and knowledge in its origins, as well as to understand the condition of novelty of the so-called experiences in the teaching of visual art. In this sense, the notion of relationship with knowledge -in the way of Beillerot and his research team at the Paris X University, coming from Lacanian psychoanalysis- gives us a greater understanding of this teaching.
\end{abstract}

Keywords: University artistic teaching, experience, relationship whit knowledge, subject, visual art

1 Profesora adjunta del Departamento de las Estéticas, Instituto Escuela Nacional de Bellas Artes, Udelar. Magíster en Psicología y Educación (Facultad de Psicología, Udelar), licenciada en Artes Plásticas y Visuales (ienba, Udelar) y licenciada en Psicología (Facultad de Psicología, Udelar). Doctoranda en Educación (FHCE, UdelaR)

2 Profesora titular en el Departamento de Enseñanza y Aprendizaje, Instituto de Educación, FHCE, Udelar. Doctora por la Universidad Complutense de Madrid, magíster en Psicología y Educación, licenciada en Psicología (Facultad de Psicología, Udelar) y licenciada en Lingüística (FHCE, Udelar). Psicoanalista, miembro de la Ecole Lacanienne de Psychanalyse. 


\section{Introducción}

El campo histórico de la investigación de la educación artística cuenta con estudios sistematizados de los modelos pedagógicos de arte visual en los distintos niveles de la enseñanza, tal como se puede observar en los trabajos de Efland $(2002,2003)$ y Agirre (2005, 2006). A este respecto, Agirre (2006) señala un modelo vigente dominante - ubicado mayoritariamente en el nivel universitario- de carácter productivo, cuyo valor está puesto en el objeto como cualidad material, calidad técnica y formal, y que se recuesta en una visión psicologicista de sujeto de aprendizaje, dejando de lado otros aspectos como el proceso y la contingencia. Dicho modelo se confirma al estudiar las últimas investigaciones publicadas sobre enseñanza artística universitaria (Huertas, 2010; Marín Viadel, 2011; Larregle, 2018; entre otras).

En nuestro país, la enseñanza artística universitaria, desde sus inicios en los años sesenta, se desarrolló en el marco de un modelo productivo basado en las pedagogías de las escuelas experimentales locales, en oposición a la enseñanza academicista del arte, y con la atención puesta en las concepciones estereotipadas del arte de los estudiantes, que remitían al academicismo. Estos estereotipos eran vistos como obstáculos para el libre desarrollo de un campo de investigación artística personal, en su vastedad y riqueza. De este modo, se consolida una enseñanza activa que se apoya en una concepción de sujeto psicologicista, cuya base teórica se compone de elementos provenientes del pragmatismo y del conductismo.

En este marco, para el primer año de la carrera, se originaron actividades experimentales que apuntaron a exponer dichas concepciones estereotipadas y que estaban orientadas por premisas, inspiradas, gran parte de ellas, en la enseñanza bauhausiana del diseño, a fin de que los estudiantes descubrieran de manera personal la vastedad del campo artístico. Estas últimas se llamaron coloquialmente experiencias; si bien no mitigaron los prejuicios acerca del arte, integraron a la enseñanza artística local una dimensión inédita que articula el proceso productivo y el saber que allí emerge. Y es la relación con el saber, en su concepción original psicoanalítica, la noción que nos lleva a comprender dicha dimensión.

\section{Enseñanza activa}

La enseñanza artística universitaria en el Uruguay se inicia en 1960 con la inscripción de la Escuela Nacional de Bellas Artes (ENBA) en la Universidad de la República, hoy Instituto Escuela Nacional de Bellas Artes (IENBA). Su fundamento pedagógico, aún vigente en los planes de estudios, se generó en defensa de un pluralismo estético y pedagógico, y en contra del modelo predominante, academicista, que provenía de la Academia de Bellas Artes francesa y que se dictaba en la Escuela, perteneciente a la órbita ministerial (Pastorino y Umpiérrez, 2007). 
Jorge Errandonea, profesor y exdirector, fue el principal ideólogo con sus «aportes doctrinarios». Estos se forjaron en la articulación entre sus ideas libertarias, el reconocimiento al método de la escuela experimental —establecido por el maestro Sabas Olaizola en una escuela de Las Piedras-, el estudio de las metodologías de la enseñanza del diseño - Bauhaus, la Escuela de Ulm- y las ideas filosóficas de Herbert Read, Erich Fromm y John Dewey, entre otros. En este marco se formula un sujeto, el educando, que es motor y protagonista de la enseñanza (Pastorino, 2016).

Los antecedentes más cercanos de esta enseñanza vienen del campo del diseño moderno (Bauhaus, Escuela de Ulm) del que Errandonea (1963) toma algunos lineamientos para realizar actividades, pero la base fundamental es la que se establece a partir de la propuesta educativa de las escuelas experimentales. Esto último condice con la afirmación que realiza Behares (2011) sobre la enseñanza universitaria local, al sostener que, a partir de la década del cincuenta del siglo pasado, «los esquemas de la educación básica» fueron tomados como modelo (p. 79). Y, si bien dicho modelo era impropio para el nivel de enseñanza superior, se trataba de un modo de respuesta de los universitarios sesentistas que, a favor de una concepción reformista de la universidad, se enfrentaban a una tradición que hacía énfasis en la producción científica y humanística, más que en los problemas de la enseñanza, y cuya matrícula no demandaba atención al respecto (Behares, 2011).

Ahora bien, en el texto inaugural de Errandonea (1963), sobre el Plan de estudios de 1960, encontramos concepciones de saber, de sujeto y de enseñanza. El saber estaba ligado a la experiencia, definida en clave deweyana, mientras que la enseñanza se fundamentaba en un método, de carácter empírico, inspirado particularmente en las escuelas experimentales locales como mencionamos antes. Así, se define un campo de aprendizaje basado en una concepción de sujeto que se conserva en el plan de 1966, en documentos anteriores al cierre del establecimiento en dictadura (Escuela Nacional de Bellas Artes [ENBA], 1970), en la reapertura democrática (ENBA, 1986; ENBA, 1991) y continúa vigente en los planes actuales. Este sujeto es el individuo con capacidades «sensibles», de imaginación y creación, el protagonista de la enseñanza gracias a la mediación de los mecanismos de la enseñanza activa. Esto correspondería, al decir de Behares (2016), a un «ego patrocinante» de la enseñanza y a un modelo conductista de aprendizaje.

Asimismo, Errandonea (1963) distingue el saber de la información. El primero implica una comprensión (por apropiación) del sujeto, producto de una experiencia personal, "es decir de un hecho vivido y sentido, reelaborado y asimilado en nuestro interior» (ENBA, 1970, p. 20). Al definir la cultura como una «matriz de desarrollo» (Errandonea, 1963, p. 84), afirma que el fin de la educación es situar en ella al educando. Lo cual exige «recrear experiencias básicas, únicas, capaces de ubicar al joven en el verdadero foco de su cultura» (Errandonea, 1963, p. 85), implicando al estudiante en una relación cultural problemática, que es válida dentro de ciertos límites, y que 
marca líneas de referencias en el campo del saber y del esfuerzo creativo. Pero también es una relación abierta a su acción ulterior.

Estas consideraciones sobre la cultura y la educación fundamentan lo que Errandonea (1963) llamó metodología empírica, la cual busca «hacerle transitar [al estudiante] las experiencias básicas que le descubren toda la perspectiva de su campo, fuera de cualquier ejercicio concreto sobre él» (p. 85).

De forma metafórica se marca la inquietud docente por la situación del estudiante que ingresa a la ENBA «venido de la calle» (p. 91), envuelto en una «corteza prejuicial» con respecto al arte que lo inhibe y limita. Según este autor, esa corteza correspondería a la versión vulgar del arte en sus aspectos visibles y convencionales, que atrae al estudiante y configura defensas y pautas rituales. Por ello, afirma que la tarea docente se centra en «medirla [a la corteza prejuicial] para hacerla saltar» (p. 86), y esto exige una técnica que produzca impacto, pues «el estudiante debe ser rudamente impactado por la extrema riqueza de una realidad primaria, que lo suma - experimentalmente- en el desconcierto de su abundancia» (p. 86). Así, deben «saltar todos los parcelamientos que fraccionaban el terreno en función de aventuras ajenas, y el estudiante, ya sin anteojeras, dispondrá de una perspectiva completa» (p. 91). Advierte, además, que las experiencias deben ser múltiples, elementales, pero espectaculares; porque de ese modo el estudiante toma contacto directo con el fondo problemático en vez de asumir una referencia externa y deformada.

Ahora bien, en los planes de estudio de 1960 (ENBA, 1960) y 1966 (ENBA, 1966), dicha técnica se sitúa en el primer año del primer período, de contenido experimental, y por su mediación le revela al estudiante un campo propio de experimentación con respecto al dibujo, color, luz, forma, etc. De esta manera, al finalizar el recorrido de este primer período, el estudiante debería adquirir el conocimiento intelectual y emocional de su campo.

En la reapertura de la ENBA en 1985, la reflexión docente sobre el impacto de la técnica en el primer año originó el ajuste de la realización de experiencias espectaculares que se habían hecho antes del cierre. Un ejemplo de esto es la vinculada al centro de interés llamado materia (ENBA, 1986) en la que se invitaba al estudiante - y se lo guiaba- a recorrer un camino con los ojos vendados y descalzo, organizado por sectores con texturas contrastadas (duras y blandas, cálidas y frías, lisas y rugosas) y marcado con un tiempo específico para cada sector con el fin de asegurar la diferenciación. El análisis que se buscaba establecer se orientaba a la cuestión de la percepción y al uso de la materia como elemento plástico en artistas del siglo xx. No obstante, según conversaciones con estudiantes en años posteriores, se observó que el impacto de la actividad desbordaba el centro de interés y su análisis; era de por sí un acontecimiento que generaba expectativas hacia las siguientes actividades en el curso. A su vez, las experiencias se popularizaron al ser realizadas en el marco de la extensión universitaria. Un ejemplo de esto es la que se realizó en el espacio municipal Centro 
de Exposiciones Subte que fue acompañada de un catálogo (ENBA, 1970). Durante los primeros años posteriores a la reapertura, según cuentan algunos docentes, la alta matriculación en el primer año se asociaba al apoyo popular de la reapertura de la ENBA y a la motivación de realizar experiencias.

Ahora bien, podemos sostener con Agamben (2001) que, si bien las experiencias se enmarcan en la concepción epistemológica que le subyace al sujeto filosófico moderno y sus efectos en el campo de la psicología, en la actualidad de la enseñanza artística universitaria su estatuto es cuestionable. Nos señala un modelo de enseñanza basada en un sujeto psicológico, cuya conducta puede ser modificada por las actividades que intervienen sobre ella. No obstante, lo que resulta de dichas experiencias es otra cosa; se trata de una vivencia fuertemente estética, atractiva y espectacular, de carácter escenográfico y lúdico, y, en algunos casos, festivo. A partir de ellas se establece cierta visibilidad del proceso personal del estudiante. En este sentido consideramos que la noción relación con el saber, en particular la concepción que forja el equipo de Beillerot (Beillerot, Blanchard-Laville, Mosconi, 1998; Beillerot et al., 2000), echa luz sobre la condición de esta situación de enseñanza.

\section{Relación con el saber y enseñanza}

Una de las líneas que estableció la noción relación con el saber en el campo de la investigación educativa fue la del Centre de Recherche Education et Formation, de la Universidad París X (CREF) liderado por Jacky Beillerot (Vercellino, 2015; Cavalcanti, 2020). En breves palabras, Beillerot et al. (1998) establece que el saber es un proceso que implica a un sujeto de deseo ( $y$, por lo tanto, incompleto, inacabado), en la dinámica del saber y no saber, y a un proceso creador constituyente de la subjetivación.

Para arribar a tal definición, parte de la noción de saberes definida en la línea arqueológica foucaultiana como el conjunto de elementos formados regularmente por la práctica discursiva. Esta definición involucra la relación de la lengua y las acciones en el campo de una práctica social, así se configura una relación saber-poder que divide y organiza las prácticas individuales y sociales (Beillerot et al., 1998). Dichos saberes son contingentes, históricamente producidos, y designan el conjunto de conocimientos en contextos sociales contradictorios, organizados en función de normas y de lógicas técnicas e ideológicas. En esta vía, Beillerot et al. (1998) señala que la arqueología foucaultiana, en pos de comprender el saber en su extensión, omite al sujeto en su relación. Por lo que incluir la cuestión del sujeto lleva necesariamente a concebir el saber como relación y proceso, subjetivo y subjetivante, que involucra la construcción psíquica en la complejidad del sujeto.

La línea de Beillerot reconoce el origen de la noción relación con el saber en un sintagma empleado en la obra lacaniana que apoya el desarrollo de las concepciones de sujeto y de saber en el psicoanálisis. Vercellino (2015) y Cavalcanti (2020) señalan 
su lugar en dos comunicaciones realizadas por Lacan en la década del sesenta: la que presentó en Royaumont «Subversión del sujeto y dialéctica del deseo en el inconsciente freudiano» (Lacan, 2002) y la lección de apertura del seminario sobre el objeto del psicoanálisis, en l'École Normale Supérieure, realizada entre 1965 y 1966, que se encuentra publicada como «La ciencia y la verdad» (Lacan, 2002).

Lacan (2002) postula un sujeto descentrado, el sujeto hablante, que, como efecto de la prematurez de su nacimiento y su total dependencia con respecto al Otro, se constituye en el resultado de una operación de lenguaje en articulación con el deseo. Esto implica que su demanda de amor y reconocimiento se inscriba en el campo de la palabra y el lenguaje. Dicho campo es exterior al sujeto y se encuentra en el campo del Otro, el cual provee los significantes, de allí que en esta concepción no quepa una interioridad, es decir, una subjetividad.

Asimismo, encuentra en la lingüística moderna y en la recapitulación de la retórica un modelo para exponer sus postulados, como se puede observar con respecto al uso del término significante. En el nivel del inconsciente -que funge como lenguaje, pero no como sistema de representación- el significante no se encuentra enlazado al significado y tiene la capacidad de establecer cadenas de significantes y efectos de sentido que interfieren en los cortes del discurso efectivo, por repetición e insistencia, produciendo traspiés en el discurso del hablante (Lacan, 2002).

Con respecto a la constitución del sujeto, el psicoanálisis muestra, entre otras tantas cuestiones, la condición pulsional de este. Ahora bien, para que advenga el deseo de saber es necesario que antes exista el deseo anudado al deseo del Otro, porque es en ese lazo donde se aloja el deseo de saber y es esto lo que confirma la escisión.

De este modo, el inconsciente, que opera para constituir al sujeto, es independiente a la conciencia. Y esta no es más que una función, una captura imaginaria del yo por su reflejo especular, que lleva al desconocimiento y a la promoción engañosa de una conciencia esencial al sujeto, que deja trasparentar el yo [je] en acto.

El sujeto postulado se basa en el sujeto de la ciencia moderna, el cartesiano, el que adviene en el cogito, con la división de la verdad y el saber (Lacan, 2002), por lo que verdad y saber no se corresponden (Fernández Caraballo, 2021). El cogito define un momento histórico por el que se liga el sujeto con el ser. Se trata de un sujeto originado en la búsqueda de la certeza y la distinción de lo verdadero y lo falso de las acciones. Y este camino conduce a rechazar todo saber, es decir, alejar de sí la experiencia y borrar la historia (Lacan, 2002).

Al revisar los aspectos problemáticos que encierra la sentencia cartesiana «pienso, luego soy», Lacan (2002) desmonta la interioridad del sujeto, arribando a que «el pensamiento no funda el ser sino anudándose en la palabra donde toda operación toca a la esencia del lenguaje» (p. 821). La causa del hablante, que para Descar- 
tes implicaba a un creador, una comunidad de ego y Dios, en la obra freudiana es el inconsciente visto como lenguaje, en la existencia de una cosa innombrable que llamará objeto a (p. 772). Por lo que, la verdad externa a la conciencia habla y es reprimida por su valor extremo (p. 823). Es así que el significante actúa e incide separado de su significación y es la causa material del sujeto hablante, pues «el efecto de lenguaje es la causa introducida en el sujeto [y gracias] a ese efecto, no es causa de sí mismo» (p. 794).

De este modo derivamos que Lacan plantea el sintagma relación con el saber a condición de postular un sujeto del deseo, que no es otro que el del cogito. Pero entendido como un momento desvaneciente de una relación con el saber anudado a una verdad que debe reprimirse, y que desarma el sentido moderno de experiencia y de conducta.

Para Beillerot et al. (1998) dicho sintagma, trasladado al campo de la investigación en educación, permite organizar interrogantes y búsquedas, y también designar y describir fenómenos. Como tal, señala el derrotero de la construcción singular del saber de un cierto sujeto, un sujeto deseante de saber (y no saber). Y, por ende, al poner en escena el deseo de saber, marca la singularidad del proceso subjetivante que se constituye en una relación dinámica y creadora. En esta vía, dicha noción permite visualizar la consistencia del psiquismo.

Es importante señalar que para el investigador no es posible retener la experiencia que comunica la vivencia, pues es efímera e intransmisible, solo se conocen las respuestas que el sujeto quiera dar (Beillerot et al., 1998). Sin embargo, se trata de una noción hablante, porque al echar luz sobre la intimidad del sujeto en relación con el saber, invita a que este reorganice sucesivamente lo que sabe, se actualice en función de ello, cuente historias y analice los resultados de tal relación. De este modo pone en evidencia el proceso creador que, como tal, tiene efecto de objetivación, acompaña la necesidad de analizar la posición propia y da lugar a la creación permanente y singular de saber sobre sí y lo real. De esta manera, la relación con el saber se da en la intimidad del sujeto, jugada entre el saber inconsciente y el que se sabe, en correlato con las fuerzas, represiones y fantasías que se ponen en movimiento. Esto es, se trata de un proceso en el que se es en relación. Por lo dicho, es el proyecto de un sujeto autor, autentificador de los saberes - que sabe que no sabe, que sabe que sabe sin saberlo, que sabe que quiere saber-y que pone en juego el valor social del saber disponible. Asimismo, Beillerot et al. (2000) distingue el aprender del saber, el aprender es producto de una relación con el dogma y las afiliaciones del sujeto. Supone, por un lado, que el sujeto acepte no saber y también que se subordine a las exigencias que imponen las cosas que deben aprenderse; y, por el otro, que acepte las respuestas que los saberes brindan, reconocer los conocimientos aceptados y las legitimaciones compartidas. Por ello, el aprender «antes de ser operatorio, exteriorizado en conductas y actos diversos, es una relación con el saber no comparable a una forma general de una relación con cualquier objeto» (p. 7). 


\section{Referencias bibliográficas}

Agamben, G. (2001). Infancia e historia: ensayo sobre la destrucción de la experiencia. Adriana Hidalgo Editora.

Agirre, I. (2005). Teorías y prácticas en educación artística: Ideas para una revisión pragmatista de la experiencia estética. Octaedro.

Aguirre, I. (2006). Modelos formativos en educación artística: imaginando nuevas presencias para las artes en educación. Universidad Pública de Navarra. Bogotá, julio de 2006. Recuperado a partir de: https://bitacoramaedar.files.wordpress. com/2013/08/imanol-aguirre-modelos-formativos-en-e-artc3adstica.pdf

Behares, L. E. (2016). La caracterización de la enseñanza como conjunto de técnicas para intervenir en los aprendizajes: un análisis crítico. Educação. Revista do Centro de Educação, 41(3). Universidade Federal de Santa Maria. http://www.redalyc.org/ articulo.oa?id=117149982008

Behares, L. E. (2011). Enseñanza y producción de conocimiento: la noción de enseñanza en las políticas universitarias uruguayas. Departamento de Publicaciones de la Universidad de la República.

Beillerot, J., Blanchard-Laville, C. y Mosconi, N. (1998). Saber y relación con el saber. Paidós.

Beillerot, J. et al. (2000). Formes et formations du rapport au savoir. L'Harmattan.

Cavalcanti, J. D. (2020). Le rapport au savoir: émergence, propagation et institutionnalisation en tant que notion dans les domaines des Sciences de l'Éducation et des Didactiques. Academia, O(19), 27-52. https://doi.org/10.26220/aca.3265

Efland, A. (2002). Una historia de la educación del arte: tendencias intelectuales y sociales en la enseñanza de las artes visuales. Paidós.

Efland, A., Freedman, K. y Stuh, P. (2003). La educación en el arte posmoderno. Paidós. Escuela Nacional de Bellas Artes (1960). Plan de estudios. ENBA.

Escuela Nacional de Bellas Artes (1966). Plan de estudios con modificaciones. ENBA.

Escuela Nacional de Bellas Artes (1970). Una experiencia educacional. 1960-1970. IMCO.

Escuela Nacional de Bellas Artes (1986). Bellas Artes: proyección de su experiencia educacional. [Catálogo]. Udelar, ENBA.

Escuela Nacional de Bellas Artes (1991). Exposición de motivos y Plan de estudios. ENBA.

Errandonea, J. (1963). El Plan nuevo de Bellas Artes. Tribuna Universitaria,11, 80-96. https://anaforas.fic.edu.uy/jspui/handle/123456789/3175

Fernández Caraballo, A. M. (2021). La relación con el saber en el discurso y en la enseñanza universitaria. S. Vercellino, D. Cavalcanti y C. Xypas (Org.) Investigaciones sobre la relación con el saber desde las Américas. Editoriales de la Universidad Nacional de Río Negro, Argentina, y de la Universidad Federal de Pernambuco, Brasil, (en prensa).

Huertas, M. (2010). Reflexiones sobre la educación artística y el debate disciplinar en Colombia. Revista Educación y Pedagogía, 22(58), 165-176. https://revistas.udea. edu.co/index.php/revistaeyp/article/view/9743/8962 
Lacan, J. (1960). Subversión del sujeto y dialéctica del deseo en el inconsciente freudiano. En J. Lacan (2002). Escritos II. Siglo XXI.

Lacan, J. (1965-1966). La ciencia y la verdad. En J. Lacan (2002). Escritos II. Siglo $\mathrm{XXI}$.

Larregle, M. (2018). Enseñar arte desde la universidad. Trayectorias Universitarias, 4(7), 32-38. https://revistas.unlp.edu.ar/TrayectoriasUniversitarias/article/ view/6367/5465

Marín Viadel, R. (2011). Las investigaciones en educación artística y las metodologías artísticas de investigación en educación: temas, tendencias y miradas. Revista Educação, 34(3), 271-285. https://www.redalyc.org/pdf/848/84820027003.pdf

Pastorino, M. (2016). A la huella de Giordano Bruno. Una arqueología del arte contemporáneo en las prácticas docentes de la ENBA (1985-1993). Tradinco.

Pastorino, M. y Umpiérrez, R. (2007). Orígenes del Plan de estudios, ENBA 1960. Biblioteca Plural, CSIC.

Vercellino, S. (2015). Revisión bibliográfica sobre la «relación con el saber». Desplazamientos teóricos y posibilidades para el análisis psicopedagógico de los aprendizajes escolares. Revista Electrónica Educare, 19(2), 53-82. 